\title{
BASIC SCIENCE ARTICLE OPEN Neuroprotective effects of Sonic hedgehog agonist SAG in a rat model of neonatal stroke
}

\author{
Vien Nguyen $\mathbb{D}^{1,2}$, Manideep Chavali ${ }^{1,2}$, Amara Larpthaveesarp ${ }^{1}$, Srikirti Kodali ${ }^{3}$, Ginez Gonzalez ${ }^{3}$, Robin J. M. Franklin ${ }^{3}$, \\ David H. Rowitch ${ }^{1,2,3,4}$ and Fernando Gonzalez ${ }^{1}$
}

BACKGROUND: Neonatal stroke affects 1 in 2800 live births and is a major cause of neurological injury. The Sonic hedgehog (Shh) signaling pathway is critical for central nervous system (CNS) development and has neuroprotective and reparative effects in different CNS injury models. Previous studies have demonstrated beneficial effects of small molecule Shh-Smoothened agonist (SAG) against neonatal cerebellar injury and it improves Down syndrome-related brain structural deficits in mice. Here we investigated SAG neuroprotection in rat models of neonatal ischemia-reperfusion (stroke) and adult focal white matter injury. METHODS: We used transient middle cerebral artery occlusion at P10 and ethidium bromide (EB) injection in adult rats to induce damage. Following surgery and SAG or vehicle treatment, we analyzed tissue loss, cell proliferation and fate, and behavioral outcome.

RESULTS: We report that a single dose of SAG administered following neonatal stroke preserved brain volume, reduced gliosis, enhanced oligodendrocyte progenitor cell (OPC) and EC proliferation, and resulted in long-term cognitive improvement. Singledose SAG also promoted proliferation of OPCs following focal demyelination in the adult rat.

CONCLUSIONS: These findings indicate benefit of one-time SAG treatment post insult in reducing brain injury and improving behavioral outcome after experimental neonatal stroke.

Pediatric Research (2021) 90:1161-1170; https://doi.org/10.1038/s41390-021-01408-7

\section{IMPACT:}

- A one-time dose of small molecule Sonic hedgehog agonist protected against neonatal stroke and improved long-term behavioral outcomes in a rat model.

- This study extends the use of Sonic hedgehog in treating developing brain injury, previously shown in animal models of Down syndrome and cerebellar injury.

- Sonic hedgehog agonist is one of the most promising therapies in treating neonatal stroke thanks to its safety profile and low dosage.

\section{INTRODUCTION}

Neonatal stroke (NS) results from large cerebral vessel disease and is an important cause of morbidity comprising long-term motor and/or cognitive deficits. ${ }^{1}$ With an incidence of 1 in 2800 live births, NS is more common than adult large vessel stroke. Secondary injury following arterial ischemia and reperfusion can lead to cell death, oxidative stress, inflammation, and altered cell proliferation and fate. ${ }^{2}$ Cerebral white matter is also highly vulnerable to ischemic injury during the neonatal period, resulting in significant functional impairment, ${ }^{3}$ which in part reflects adverse effects on oligodendrocyte lineage cells. Indeed, oligodendrocyte progenitor cells (OPCs) continue to undergo significant changes during the perinatal and postnatal period and are vulnerable to ischemic injury. ${ }^{4}$ Currently, we lack neuroprotective therapies for NS.

Sonic hedgehog (Shh) signaling plays a crucial role in early central nervous system (CNS) development, influencing oligodendrocyte development. ${ }^{5}$ Shh is a secreted protein that functions by inhibiting the transmembrane receptor Patched and inducing activity of Smoothened, allowing for activation of downstream pathway targets, including Gli1 and Cyclin D1 (CCD1). The cilium is present on neural stem cells and has recently been shown to be present in OPCs but absent in mature oligodendrocytes, corresponding to loss of responsiveness to Shh. ${ }^{5}$ In addition, astroglial-secreted Shh has been shown to play a role in blood-brain barrier (BBB) maturation and maintenance, along with post-insult anti-inflammatory effects. $^{6}$

SAG is a small molecule agonist of the Smo-Shh pathway. We have previously demonstrated efficacy of SAG treatment in a mouse model of neonatal cerebellar injury. ${ }^{7}$ In addition, SAG treatment starting at birth has also been found to be beneficial in a mouse model of Down syndrome. ${ }^{8}$ However, these models are

\footnotetext{
${ }^{1}$ Department of Pediatrics, University of California San Francisco, San Francisco, CA, USA; ${ }^{2}$ Eli and Edyth Broad Center for Stem Cell Research and Regenerative Medicine,

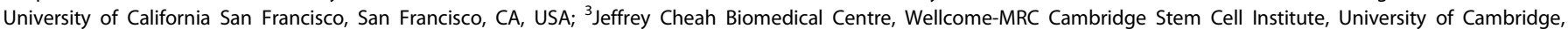
Cambridge, UK and ${ }^{4}$ Department of Paediatrics, University of Cambridge, Cambridge, UK

Correspondence: David H. Rowitch (Dhr25@medschl.cam.ac.uk) or Fernando Gonzalez (Fernando.Gonzalez@ucsf.edu)
}

Received: 26 October 2020 Revised: 20 January 2021 Accepted: 27 January 2021

Published online: 2 March 2021 
distinct from the forebrain injury commonly seen in human preterm and full-term neonates.

We have previously described a non-hemorrhagic ischemia-reperfusion focal stroke model in the P10 immature rat using transient unilateral middle cerebral artery occlusion (tMCAO). ${ }^{9}$ tMCAO mimics large vessel NS and is thus suitable for testing pathobiology and post-injury treatment strategies. For instance, we have described alterations in neural precursor cell fate following tMCAO that affects both the subcortical white matter and cortical/subcortical gray matter. ${ }^{9}$ Oligodendrocyte injury leads to loss of myelin sheaths, which are important for metabolic support and transmission of action potentials. ${ }^{10}$ Oligodendrocyte maturation arrest is observed in neonatal white matter injury. ${ }^{11}$ Therefore, enhancing and optimizing the endogenous oligodendrocyte response could improve long-term outcomes. To further elucidate oligodendrocyte biology following demyelinating injuries, we used the adult cerebellar peduncle focal demyelination model as it has been well characterized. ${ }^{12}$

There has been a significant focus on developing therapeutic strategies using biologics to enhance endogenous repair processes following brain injury. Recent studies have shown preserved neuronal numbers, angiogenesis, and improved outcomes following Shh or SAG treatment in adult stroke models. ${ }^{13}$ Here we investigated the neuroprotective effects of SAG in two distinct models of focal brain injury: (1) neonatal unilateral MCA ischemia-reperfusion in the immature rat, and (2) focal toxin-induced demyelinating cerebellar peduncle white matter injury in the mature rat. We examined both short-term histological and long-term behavioral outcomes after early stroke and SAG-mediated cell-type-specific effects on forebrain gray and white matter parenchyma.

\section{METHODS}

All animal research was approved by the University of CaliforniaSan Francisco and University Biomedical Services of the University of Cambridge Institutional Animal Care and Use Committees and in compliance with United Kingdom Home Office regulations.

Preparation of SAG and vehicle

Synthesis of SAG was performed as described previously. ${ }^{14}$ SAG was dissolved to $5 \mathrm{mM}$ in dimethyl sulfoxide (DMSO) and then further diluted in normal saline to contain 5\% DMSO.

\section{In vivo SAG bioactivity}

SAG was injected in Gli-Luciferase reporter mice ${ }^{15}$ at a concentration of $50 \mathrm{mg} / \mathrm{kg}$ body weight. Mice were anesthetized with isoflurane and placed in an in vivo bioluminescent imaging system (Xenogen) to detect luciferase reaction. SAG bioactivity was visualized by increase in intensity in transgenic animals.

Transient middle cerebral artery occlusion

Postnatal day 10 (P10) Sprague-Dawley rats underwent focal ischemia-reperfusion with tMCAO for $3 \mathrm{~h}$ or sham surgery, as previously described. ${ }^{9}$ The right internal carotid artery (ICA) was dissected and an arteriotomy was made proximal to the isolated ICA. A silicone-coated 6-0 nylon filament was inserted 9-10.5 mm to occlude the MCA and secured for the duration of occlusion. Following induction, rectal temperature was monitored and maintained at $36-37^{\circ} \mathrm{C}$ with a combination of heating blanket and overhead light until recovery from anesthesia. For reperfusion, each animal was anesthetized, and suture ties and the occluding filament were removed. Avitene Microfibrillar Collagen Hemostat was placed over the arteriotomy and the skin incision was closed. Sham animals were anesthetized, and the ICA was dissected, after which the skin incision was closed. Immediately following reperfusion, animals received a single intraperitoneal (IP) injection of SAG $(50 \mathrm{mg} / \mathrm{kg})$ or vehicle. Weight was monitored for 1 week following tMCAO or sham surgery to ensure adequate weight gain.
Thirty-two animals underwent tMCAO, and 22 animals sham surgery (54 animals in total). Animals were split into four groups (Sham+Veh, $n=5$; Sham + SAG, $n=5$; tMCAO+Veh, $n=8$; tMCAO + SAG, $n=8$, Supplementary Fig. S1) for cell fate analysis at 2 weeks post-surgery and then were injected with bromodeoxyuridine (BrdU; $25 \mathrm{mg} / \mathrm{kg}$ twice daily) from age P11 to P15 to label newly generating cells. For the second cohort of animals, behavioral analysis was performed at 2 months of age (Sham+Veh, $n=6$; Sham+SAG, $n=6$; tMCAO+Veh, $n=8$; tMCAO+SAG, $n=8)$.

Western blot analysis and quantitative reverse transcription-PCR (qRT-PCR)

Tissue lysates were obtained from ipsilateral and contralateral cortices of Sham+Veh, Sham+SAG, tMCAO+Veh, and tMCAO + SAG $24 \mathrm{~h}$ post-reperfusion or equivalent ( $n=3$ per group). For protein analysis, fresh-frozen tissues were homogenized in RIPA buffer (Thermo Scientific) containing protease inhibitors (Roche). Immunoblotting and fluorescent detection were done as previously described using the Odyssey system (Li-Cor). ${ }^{4}$ Antibodies included CCD1 (rabbit polyclonal, Thermo), Iba1 (rabbit polyclonal, Wako), glial fibrillary acidic protein (GFAP; mouse monoclonal, Sigma), and $\beta$-Actin (mouse ascites, Sigma). For qRT-PCR, RNA was isolated with Trizol followed by RNeasy (Qiagen) from tissue lysates and assayed for Gli1 gene expression with SYBR-Green (Roche) on a LightCycler 480 (Roche).

\section{Histology}

Animal brains were harvested at either 2 weeks post-surgery or following completion of behavioral testing at 2 months, as previously described. ${ }^{16}$ The entire brain was sectioned at $50-\mu \mathrm{m}$ intervals on a sliding microtome (Thermo), and serial sections were mounted, dried, and stained with Cresyl Violet or the following antibodies: BrdU [Rat Mab BU1/75(ICR1), Accurate Chemicals], Olig2 (Mouse Mab from C.D. Stiles, Harvard), GFAP (Rat Mab 2.2B10, Thermo), Iba1 (Rabbit Pab, Wako), CC1 (APC, Mouse Mab OP80, Calbiochem), Nkx2.2 (Mouse Mab 74.5A, Developmental Studies Hybridoma Bank), Collagen 4A (Col4A; Goat Pab, AB769 Millipore), Claudin 5 (Mouse Mab, 35-2500 Invitrogen).

Focal demyelination in cerebellar white matter

Young CD rats (10-12 weeks old, Charles River) were used for the surgical procedures. Ethidium bromide (EB; $0.01 \%$ ) was injected into the rat caudal cerebellar peduncle bilaterally to induce demyelination as previously described. ${ }^{12}$ Animals in the treatment group received SAG $(50 \mathrm{mg} / \mathrm{kg})$ IP 5 days post injury (dpi) while control (sham) group received a single injection of saline IP at the same time point. OPC recruitment, proliferation, and differentiation are key for successful remyelination. To avoid the acute effects in this model on OPC proliferation and recruitment and focus on later repair, treatment was administered 5 days following EB injection as previously published. ${ }^{17,18}$ Treatment and sham animals were perfused at 7 or $14 \mathrm{dpi}$ to study histology. Frozen sections $(12 \mu \mathrm{m})$ were collected using a cryostat (Bright Instruments). Oligodendrocyte proliferation and maturation were studied at $7 \mathrm{dpi}$ (Nkx2.2, Sham+Veh, $n=4$, Sham+SAG, $n=3$; Nkx2.2/Ki67, Sham+Veh, $n=4$, Sham+SAG, $n=3$; Olig2/CC1, Sham + Veh, $n=4$, Sham + SAG, $n=4 ; \mathrm{PLP}$, Sham + Veh, $n=3$, Sham +SAG, $n=4$ ), while mature oligodendrocytes were studied at 14 dpi (Olig2/CC1, Sham+Veh, $\mathrm{n}=5$,Sham+SAG, $n=5$; PLP, Sham + Veh, $n=4$, Sham + SAG, $n=3$; Supplementary Fig. S1).

\section{In situ hybridization}

The expression of proteolipid protein (PIp) mRNA (1:2000) in demyelinated lesions was examined by in situ hybridization with digoxigenin-labeled cRNA probes on cryostat sections $(12 \mu \mathrm{m})$ using the established protocols. ${ }^{19}$ ImageJ 1.44 (Wayne Rasband) was used to determine the number of positive cells within the lesions on digitized sections. 
Behavioral testing

Cylinder Rearing (CR) was used to assess the effects of ischemic injury and SAG treatment on forelimb use as a function of sensorimotor bias, as previously described. ${ }^{20}$ Animals were handled for about 5 min per day for 3 days prior to testing, which was conducted 7.5 weeks after surgery. Each animal was then individually placed in a transparent Plexiglass cylinder $(20-\mathrm{cm}$ diameter, $30-\mathrm{cm}$ height) and observed for 3-min trial on 2 consecutive days, with results averaged per animal. Initial forepaw placement of each weight-bearing contact with the wall was recorded as right, left, or both forepaws. Results were expressed as the percentage use of the non-impaired (right) forepaw for braces relative to the total number of forepaw initiations.

Novel Object Recognition (NOR) was performed to assess recognition memory at 8 weeks following surgery. ${ }^{21}$ Habituation was performed on days 1 and 2, for a 10-min trial each day, in a $40 \mathrm{~cm} \times 40 \mathrm{~cm}$ box with $30 \mathrm{~cm}$ tall walls in a room lacking distinctive navigational cues. A 5-min familiarization trial was performed on day 3 , with two identical objects inside the testing box. Finally, a 3-min testing phase or NOR trial was performed on day $4,24 \mathrm{~h}$ following the familiarization phase, where one familiar object was replaced by a new object of the same size. The animal exploration time of the novel object was recorded: novel object preference index $(\%)=$ novel object exploration time/(NOR time + familiar object exploration time) $\times 100$.

Stereological analysis

Unbiased, systematic random sampling was used to quantify both hemisphere and corpus callosum (CC) volume. A series representing every 12th section was selected, Cresyl Violet stained, and analyzed. Sections encompassed the whole brain rostrally from the genu of the CC through the posterior portion of the hippocampus to the occipital lobes caudally. All volumetric quantifications were performed in a blinded manner on a Zeiss AxioScope Imager Z.2 with a motorized stage, with the Neurolucida and Stereoinvestigator software (MicroBrightField). For the regions of interest (ROIs), the ipsilateral (lesioned) and contralateral (control) hemispheres ( $\times 4$ magnification) and CC $(\times 10)$ were traced. The cross-sectional area of the ROI was calculated according to the Cavalieri principle, as previously published. ${ }^{22}$ Damage secondary to stroke was determined quantitatively by calculating the percentage of volume of the ipsilateral vs contralateral hemispheres.

For quantitative analysis of cell types, a systematic random sampling of every 24th coronal section (3-4 sections per animal) was single or double immunostained. Sections were imaged using a Zeiss Axiolmager.A2 upright microscope and analyzed using Stereoinvestigator. On each section, a tile scan of the entire hemisphere was imaged, the ROI (striatum or CC) was outlined, and the $\times 20$ field was randomly placed within the $\mathrm{ROI}$ for imaging. Following imaging of the full-thickness $z$ stack (1- $\mu \mathrm{m}$ steps) with $10-\mu \mathrm{m}$ border zones, the field was moved in an automated fashion resulting in imaging of approximately $18 \%$ of the ROI. Single and co-labeled cells were quantified using the Optical Fractionator probe for unbiased cell counting within the software. Cell density was calculated as average number of cells per $\times 20$ field, cell percentage was calculated as the total number of co-labeled cells per single label, and total cell number in the CC was calculated using $\mathrm{Nv} \times$ Vref calculation based on density and volume of the measured $C C$, respectively.

Statistical analysis

Data are presented as mean \pm SEM. Sex was equally distributed between groups and time points, and no differences were seen between female and male animals. All analyses were conducted by investigators blinded to the treatment group. For statistical analysis, nonparametric methods were used. The one-way analysis of variance Kruskal-Wallis test was applied for comparisons of multiple groups followed by the Wilcoxon rank-sum test for comparison between the two groups. $p$ Values were corrected by the Bonferroni method to maintain the $a$ value at 0.05 . All statistical analyses were performed using the SAS Enterprise Guide software (SAS Institute, Cary, NC, U.S.A.).

\section{RESULTS}

Single-dose SAG treatment preserves brain volume following neonatal tMCAO

We determined the optimal concentration of SAG $(50 \mathrm{mg} / \mathrm{kg})$ by dose-response in vivo using Gli-luciferase mice injected at P10 and followed for $120 \mathrm{~h}$ (Supplementary Fig. S2), where we saw elevated luciferase signal for at least $72 \mathrm{~h}$. We tested different doses in rats (10-100 mg/kg body weight, data not shown), and found that $50 \mathrm{mg} / \mathrm{kg}$ resulted in the most potent changes similar to our results in mice. To determine the efficacy of post-insult onetime SAG treatment on forebrain injury in the immature brain, we performed 3-h tMCAO or sham surgery in P10 rat pups that were then treated with SAG or vehicle at the time of reperfusion (Fig. 1a). In rats, P10 is considered a rough developmental equivalent to the full-term human neonate, when NS is most common. Prior work has shown that the tMCAO model consistently results in moderate injury involving the ipsilateral striatum, subcortical white matter, and parieto-temporal cortex. ${ }^{9}$ Both sham-operated animals that received SAG or vehicle treatment $\quad($ Sham + Veh $=0.98 \pm 0.03, \quad$ Sham + SAG $=1.01 \pm 0.04$; Fig. 1b, c) showed minimal hemispheric impact. While tMCAO +Veh animals showed consistent, significant hemispheric volume loss 2 weeks following tMCAO (tMCAO+Veh $=0.61 \pm 0.04 ; p<$ $0.001)$, single-dose SAG treatment at the time of reperfusion significantly preserved hemispheric brain volume in tMCAO animals $\quad$ tMCAO+Veh $=0.61 \pm 0.04, \quad \mathrm{tMCAO}+\mathrm{SAG}=0.82 \pm 0.03$; $p<0.01$ ), including the CC (tMCAO+Veh $=0.565 \pm 0.04$; tMCAO + SAG $=0.732 \pm 0.05, p<0.05$, Fig. $1 \mathrm{~d}$ ), almost to sham levels. Thus there is a significant neuroprotective effect of a one-time SAG treatment following a tMCAO ischemic insult.

SAG treatment activates downstream Shh targets in the ipsilateral hemisphere and reduces gliosis following neonatal tMCAO To confirm that SAG administration targets the Shh pathway, we performed protein/gene expression analysis from brain samples collected $24 \mathrm{~h}$ after reperfusion to determine the expression of downstream Shh targets CCD1 and Gli1. Both CCD1 (Fig. 2a, b) and Gli1 (Fig. 2c) showed significantly increased protein (CCD1) and gene expression (Gli1) levels in the tMCAO+SAG group (CCD1 lpsi/Contra expression ratio: $\mathrm{tMCAO}+\mathrm{Veh}=0.60 \pm 0.047$, $\mathrm{tMCAO}+\mathrm{SAG}=1.5 \pm 0.26, p<0.05 ; \mathrm{Gli} 1 \mathrm{Ipsi} /$ Contra expression ratio: $\mathrm{tMCAO}+\mathrm{Veh}=0.24 \pm 0.0014, \mathrm{tMCAO}+\mathrm{SAG}=1.1 \pm 0.17$, $p<0.05)$.

Neonatal hypoxia-ischemia injury is typically followed by widespread inflammation involving parenchymal microglia and astrocytes, which could contribute to secondary injury. ${ }^{23}$ To investigate the effects of SAG treatment on gliosis, we performed immunohistochemical analysis of activated microglia (Iba1) and reactive astrocytes (GFAP) in gray matter regions post-neonatal tMCAO. As shown (Fig. 2d, e), we observed a decrease in ipsilateral lba1 staining, which suggests decreased microglial inflammation, and astrocytosis in tMCAO+SAG compared to $\mathrm{tMCAO}+\mathrm{Veh}$ animals. When quantified by immunoblotting, both Iba1 and GFAP protein expression were significantly increased in the ipsilateral hemisphere in tMCAO +Veh compared to the sham groups but showed no significant difference when SAG was administered (Sham+Veh $=1.05 \pm$ 0.26 ; $\mathrm{tMCAO}+\mathrm{Veh}=7.58 \pm 0.59 ; \mathrm{tMCAO}+\mathrm{SAG}=1.55 \pm 0.13, p<$ 0.01 , Fig. $2 f, g)$. These findings indicate that SAG treatment induces Shh targets and may decrease inflammation and gliosis following tMCAO. 

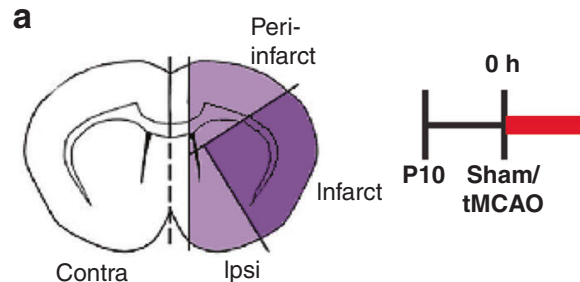

b

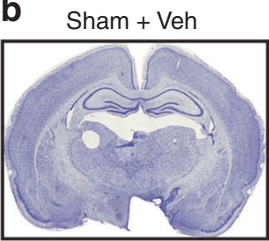

tMCAO + Veh

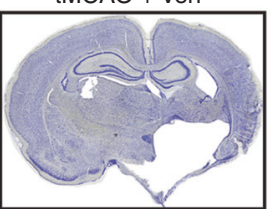

Sham + SAG

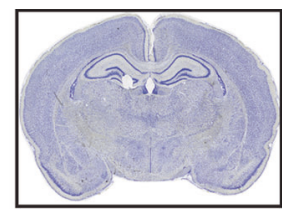

$\mathrm{tMCAO}+\mathrm{SAG}$

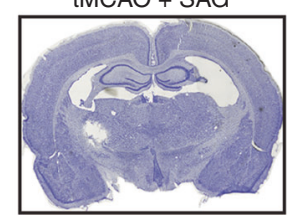

C

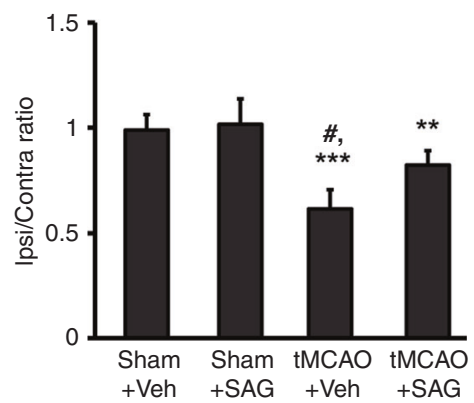

d

Corpus callosum volume

Fig. 1 SAG administration following tMCAO improves brain volume. a Diagram of transient middle cerebral artery occlusion (tMCAO) injury region in a coronal section of a rat brain, and experimental timeline for $\mathrm{TMCAO}$ and volume/cell fate analysis. Contra contralateral/uninjured hemisphere, Ipsi ipsilateral/injured hemisphere. $\mathbf{b}$ Representative images of coronal sections from rat brains 2 weeks post-tMCAO or sham surgery, at P24. c Quantification of brain volume following surgery and treatment, showing ratio of injured (ipsilateral) and uninjured (contralateral) hemispheric volumes. d Quantification of corpus callosum volume following surgery and treatment. For quantification, mean \pm SEM; ${ }^{* *} p<0.01$ and ${ }^{* * *} p<0.001$ vs Sham +Veh, ${ }^{\#} p<0.05$ vs tMCAO+SAG; one-way ANOVA with Dunnett's test. Veh injection of vehicle solution $(0.85 \%$ saline solution).

a

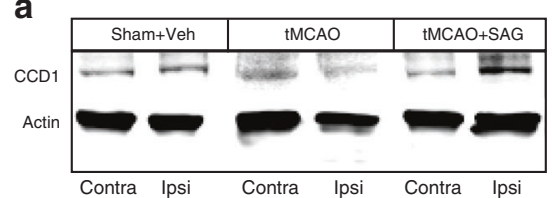

b

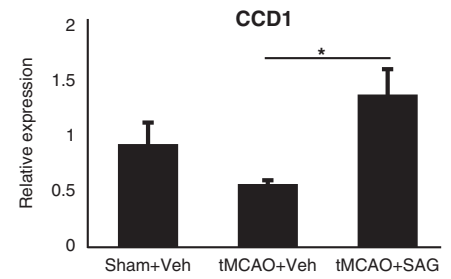

C

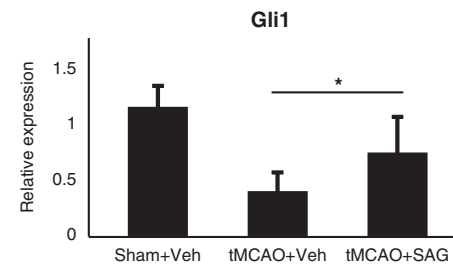

d

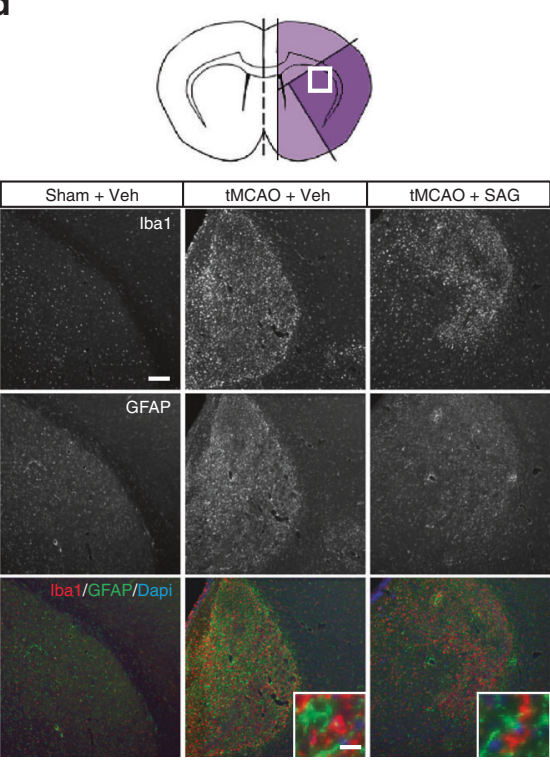

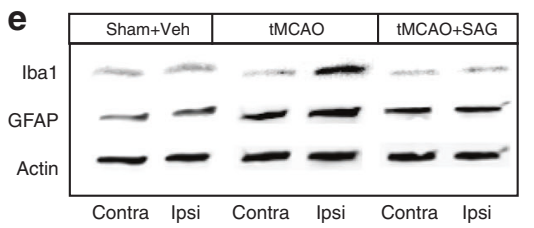

f

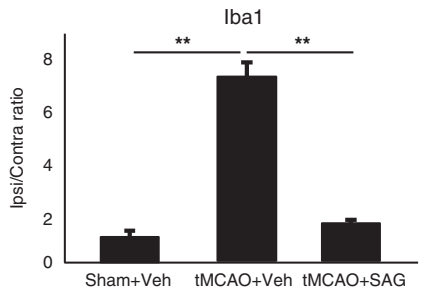

g

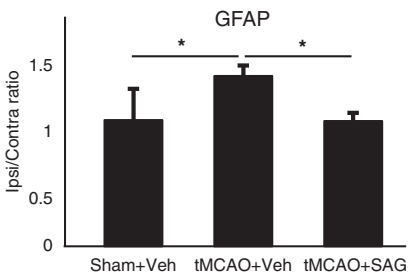

Fig. 2 SAG activates downstream Shh targets and decreases gliosis. a Brains collected $24 \mathrm{~h}$ post-surgery analyzed for protein expression of the Shh downstream target Cyclin D1 (CCD1) expression in Sham+Veh, tMCAO+Veh, or tMCAO+SAG. Contra contralateral/uninjured hemisphere, Ipsi ipsilateral/injured hemisphere. b Quantification of CCD1. c tMCAO brains collected $24 \mathrm{~h}$ post-surgery analyzed for mRNA expression of Shh downstream target Gli1 using qRT-PCR. d Diagram showing striatum from infarct region of tMCAO brain, and representative immunofluorescence images of Iba1, GFAP, or merged. Inset, higher magnification of cells positive for Iba1 and GFAP. Scale bar, $500 \mu$ m; inset, $20 \mu \mathrm{m}$. e Representative immunoblots of Iba1 and GFAP expression in Sham+Veh, tMCAO+Veh, or tMCAO+SAG brains. Contra contralateral/ uninjured hemisphere, Ipsi ipsilateral/injured hemisphere. $\mathbf{f}$ Quantification of Iba1 ipsilateral-to-contralateral expression ratio. $\mathrm{g}$ Quantification of GFAP. ${ }^{*} p<0.05 ;{ }^{* *} p<0.005$, Student's $t$ test. 
a
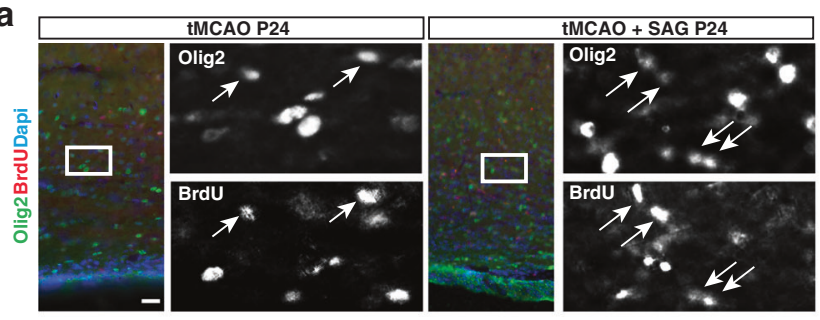

d

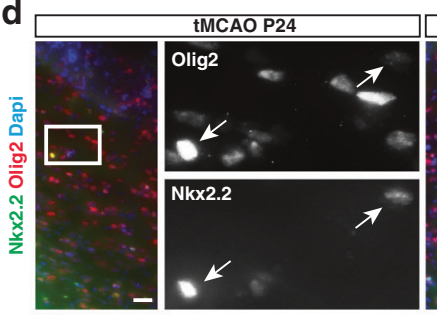

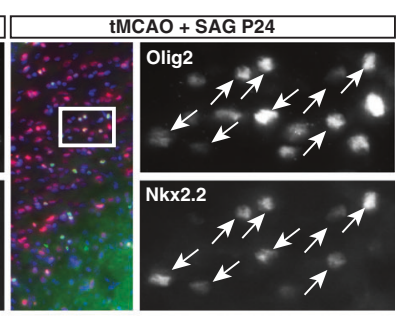

b
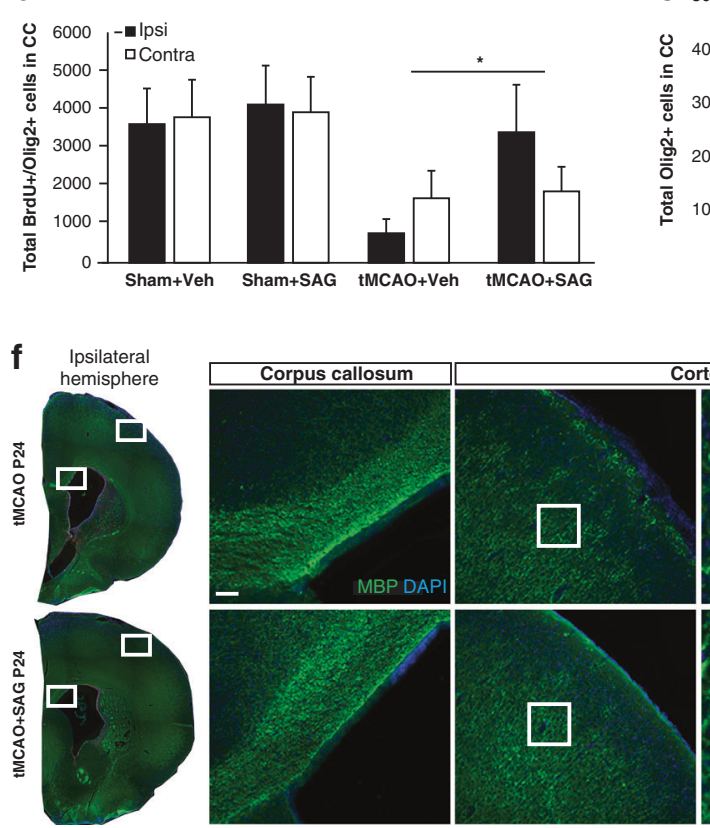

e
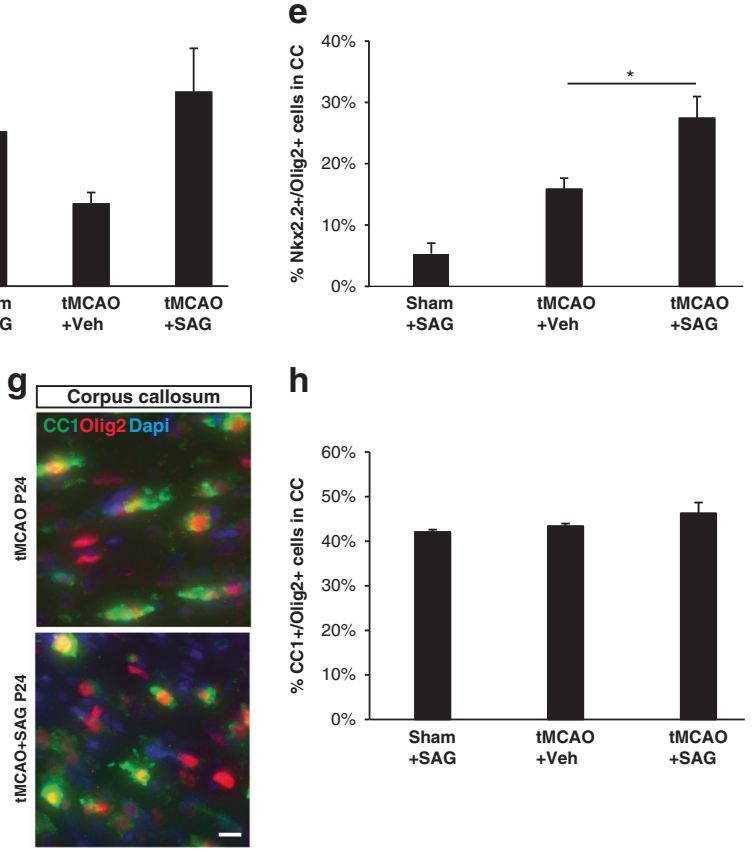

h

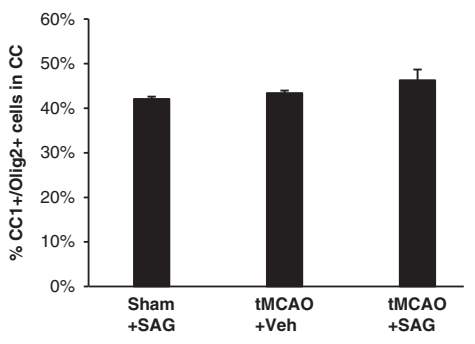

Fig. 3 SAG increases oligodendrocyte proliferation but not differentiation following tMCAO. a Representative images showing proliferating cells $(\mathrm{BrdU}+)$ in the corpus callosum (CC) positive for Olig2 in rats given tMCAO administered with saline (left) or SAG (right) at P24. Scale bar, $50 \mu \mathrm{m}$. Inset, magnified view of single-channel immunostains. Arrows denote double-labeled cells. b Quantification of cells double-labeled with BrdU and Olig2 in the CC. Black bars, CC from ipsilateral hemisphere; white bars, CC from contralateral hemisphere. c Quantification of total Olig2 + cells in the CC in the ipsilateral hemisphere. d Representative images showing Olig2+ cells double-labeled with Nkx2.2 in the CC in rats given tMCAO administered with saline (left) or SAG (right) at P24. Scale bar, $50 \mu \mathrm{m}$. Inset, magnified view of single-channel immunostains. Arrows denote double-labeled cells. e Proportion of Nkx2.2+/Olig2+ cells in the CC of the ipsilateral hemisphere in tMCAO+SAG vs tMCAO+Veh. $\mathbf{f}$ Representative images of the ipsilateral hemispheres stained for MBP (green) in P24 rats (left), and insets shown at higher magnification (right). Scale bar, $500 \mu \mathrm{m}$. g Representative images showing Olig2+ cells double-labeled with CC1 in the CC in rats given tMCAO administered with saline (top) or SAG (bottom). Scale bar, $20 \mu \mathrm{m}$. h Quantification of CC1+/Olig2+ cells in the CC of the ipsilateral hemisphere in tMCAO+SAG vs tMCAO+Veh. ${ }^{*} p<0.05$, one-way ANOVA with Tukey's multiple comparisons test.

SAG increases OPC proliferation following neonatal tMCAO and adult demyelinating injury

Our analysis that SAG could improve hemispheric brain volume preservation specifically showed improvements in the CC, which could reflect axon preservation and/or support of myelinating oligodendrocytes or OPCs. We next focused on the effects of SAG treatment on the oligodendrocyte lineage. Using Olig2 as a panlineage marker and BrdU + co-labeling, we observed a significant increase in proliferating OPCs in SAG-treated animals 14 days post-tMCAO in the ipsilateral CC (tMCAO+Veh $=769 \pm 371$ vs. $\mathrm{tMCAO}+\mathrm{SAG}=3400 \pm 1235 ; p<0.01$, Fig. 3a, b) but not total Olig2 + cells (Fig. $3 c$ ). This was associated with an increase in the proportion of NKX2.2+ OPCs in the tMCAO+SAG group compared to controls (Shams $=0.052 \pm 0.023 ; \mathrm{tMCAO}+\mathrm{Veh}=0.16 \pm 0.023$; $\mathrm{tMCAO}+\mathrm{SAG}=0.27 \pm 0.18 ; p<0.01$, Fig. $3 \mathrm{~d}$, e). However, despite increased immature oligodendrocytes in the CC of SAG-treated animals, we found that myelin basic protein labeling appeared similar to Veh-treated controls (Fig. 3f) and that co-labeled mature Olig2/CC1+ oligodendrocytes were similar in the two groups (Fig. 3g, h).
To further interrogate the SAG effects on remyelinating oligodendrocytes, we used a focal EB-induced demyelination model $^{12}$ in adult rats followed by one-time SAG or vehicle injection (Fig. 4a, b). This model is characterized by demyelination without axonal loss and has a well-established temporal sequence of remyelination involving OPC proliferation (7 dpi) and differentiation (14 dpi). The lesion area was determined by DAPI hypercellularity, and we used NKX2.2/Ki67 and Olig2/CC1 to mark proliferating OPCs and differentiated cells, respectively. As shown (Fig. 4c, d), at 7 dpi there were increased numbers of newly

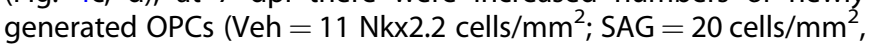
$p<0.05)$. There was an increase in the number of Olig2/CC1 at 7 dpi, which suggests a pro-differentiation effect of SAG on OPCs at early time points; there was no differences at $14 \mathrm{dpi}$ between the vehicle and treated group. Staining for the Plp, a marker of myelinforming oligodendrocyte, showed an increase of the Plp+ cells: however, this was not statistically significant (similar results were found at $14 \mathrm{dpi}$ tissue; there were no statistically significant differences between the groups). Together, these findings indicate that SAG treatment transiently increases 
a

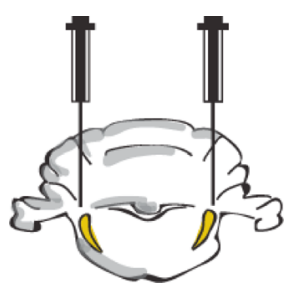

b

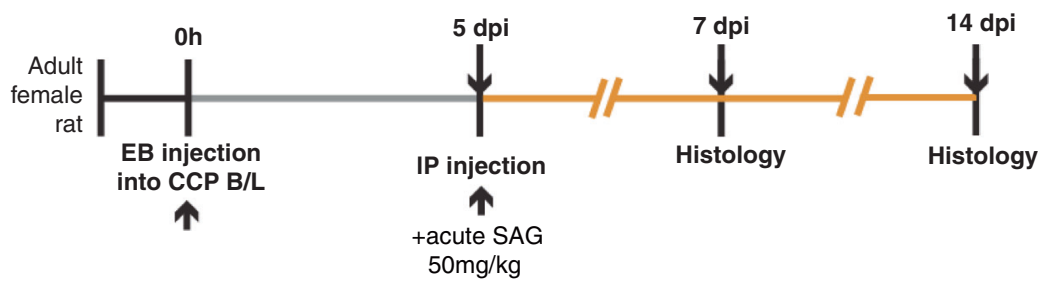

C
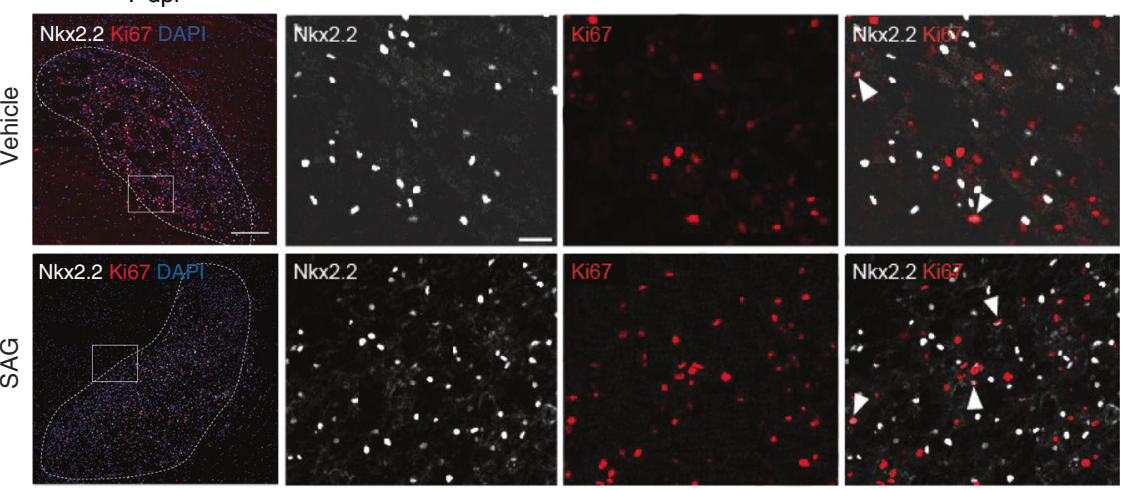

d

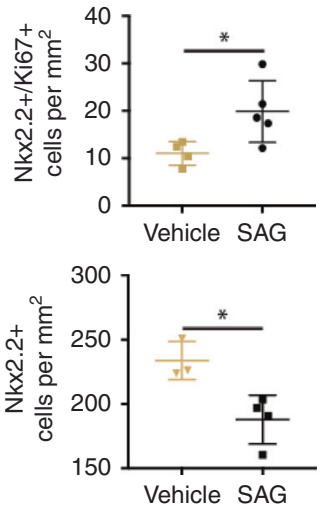

Fig. 4 SAG promotes oligodendrogenesis and maturation in an adult rat model of focal demyelination. a Schematic of EB model. b Experimental timeline for focal demyelination using ethidium bromide (EB) injection into the rat cerebellar peduncle (CCP). c Representative images of proliferating cells (Ki67, red) and OPCs (Nkx2.2, white) stained within the lesioned area (dotted lines) at 7 days post injury (dpi). Scale bar, $25 \mu \mathrm{m}$. Inset, magnified view. Scale bar, $10 \mu \mathrm{m}$. Arrowheads denote cells double-positive for Ki67 and Nkx2.2. d Quantification of Nkx2.2 +/Ki67+ cells (top), and total Nkx2.2+ cells (bottom) from lesioned areas after vehicle or SAG administration. ${ }^{*} p<0.05$, Student's $t$ test.

oligodendrocyte proliferation at early stages post-injury but has no sustained effects on differentiation and remyelination. Taken together, these findings suggest that SAG treatment results in increased CC volume and levels of OPC proliferation but not oligodendrocyte differentiation or remyelination.

SAG promotes angiogenesis following tMCAO

Angiogenesis is a universal feature of wound healing across tissues, and Shh has known effects in promoting vascular integrity. To determine whether SAG treatment promoted vascular remodeling post-injury, we co-labeled sections harvested at P24 with $\mathrm{BrdU}+$ (proliferation) and Col4A (a vascular basement membrane marker), to quantify endothelial cell (EC) proliferation in the injured core following tMCAO or sham surgery (Fig. 5a). As OPCs have been shown to play an important role in endothelial proliferation and vascular development during cerebral development ${ }^{24}$ and under hypoxic conditions, ${ }^{4}$ we also explored the possibility that an increase in OPC number is associated with angiogenesis following injury and treatment. We did observe a close association of proliferating oligodendrocytes with the vasculature in the injured striatum in tMCAO+SAG compared to tMCAO+Veh animals (Fig. 5b). Finally, immunohistochemical analysis of Claudin-5 co-labeled with Col4A indicated no significant change in expression or vascular coverage, suggesting that SAG does not regulate endothelial tight junction protein expression (Fig. 5c).

We observed a significant increase in the density of co-labeled, newly generated ECs in tMCAO+SAG animals (1067 \pm 163 cells per hpf) vs tMCAO+Veh animals $(578 \pm 154, p<0.05$; Fig. $5 \mathrm{~d}$ ), although there was no difference in vascular coverage (Fig. 5e), which could be attributed to the relatively early time point chosen for analysis. ${ }^{25}$ To determine whether endothelial proliferation contributed to increased angiogenesis, we analyzed vascular network features across cortical, striatal, and white matter regions and found a significant increase in vessel branching in
tMCAO+SAG animals, consistent with endothelial proliferation at this early time point ( $\mathrm{tMCAO}+\mathrm{SAG}=49,447 \pm 3189$, tMCAO+ $V e h=35,068 \pm 6268 ; p<0.05$; Fig. 5f). Given these findings, it is possible that SAG-induced OPC proliferation is associated with the pro-angiogenic response.

SAG improves long-term histology and cognitive function after tMCAO

To determine the long-term efficacy of single-dose SAG treatment after neonatal forebrain ischemia-reperfusion injury, we repeated the P10 tMCAO experiment and analyzed this cohort in young adulthood (Fig. 6a). At 8 weeks following tMCAO (P66), we used CR testing to assess sensorimotor function and NOR as a test of cognitive function and recognition memory. Brains were then harvested for volume analysis (P72). As shown (Fig. 6b), while there was no significant improvement in sensorimotor function after SAG treatment, we observed a significant improvement in cognitive function and recognition memory as assessed by NOR (Fig. 6c). There was a significant decrease in the percentage of novel object interaction in tMCAO+Veh compared to tMCAO + SAG animals, which did not differ from shams (tMCAO+Veh $=$ $0.44 \pm 0.04, \quad \mathrm{tMCAO}+\mathrm{SAG}=0.58 \pm 0.03, \quad p<0.05$ ). Finally, we observed a persistent improvement in hemispheric brain volume at this later time point with SAG treatment $(\mathrm{tMCAO}+\mathrm{SAG}=0.56 \pm$ $0.14, \mathrm{tMCAO}+\mathrm{Veh}=0.73 \pm 0.12, p<0.05$; Fig. 6d), along with improved CC volume (tMCAO+Veh $=0.52 \pm 0.06, \mathrm{tMCAO}+\mathrm{SAG}=$ $0.79 \pm 0.06, p<0.05 ;$ Fig. 6e, f).

\section{DISCUSSION}

In normal development, the Shh signaling pathway is crucial for CNS patterning and cell fate specification, ${ }^{26}$ proliferation of cerebellar granule neuron precursors, ${ }^{27}$ neural circuit and synapse formation, ${ }^{28}$ vascular development, ${ }^{29}$ and oligodendrogenesis. ${ }^{30}$ We and others have shown benefits of Shh pathway activation in 


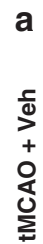
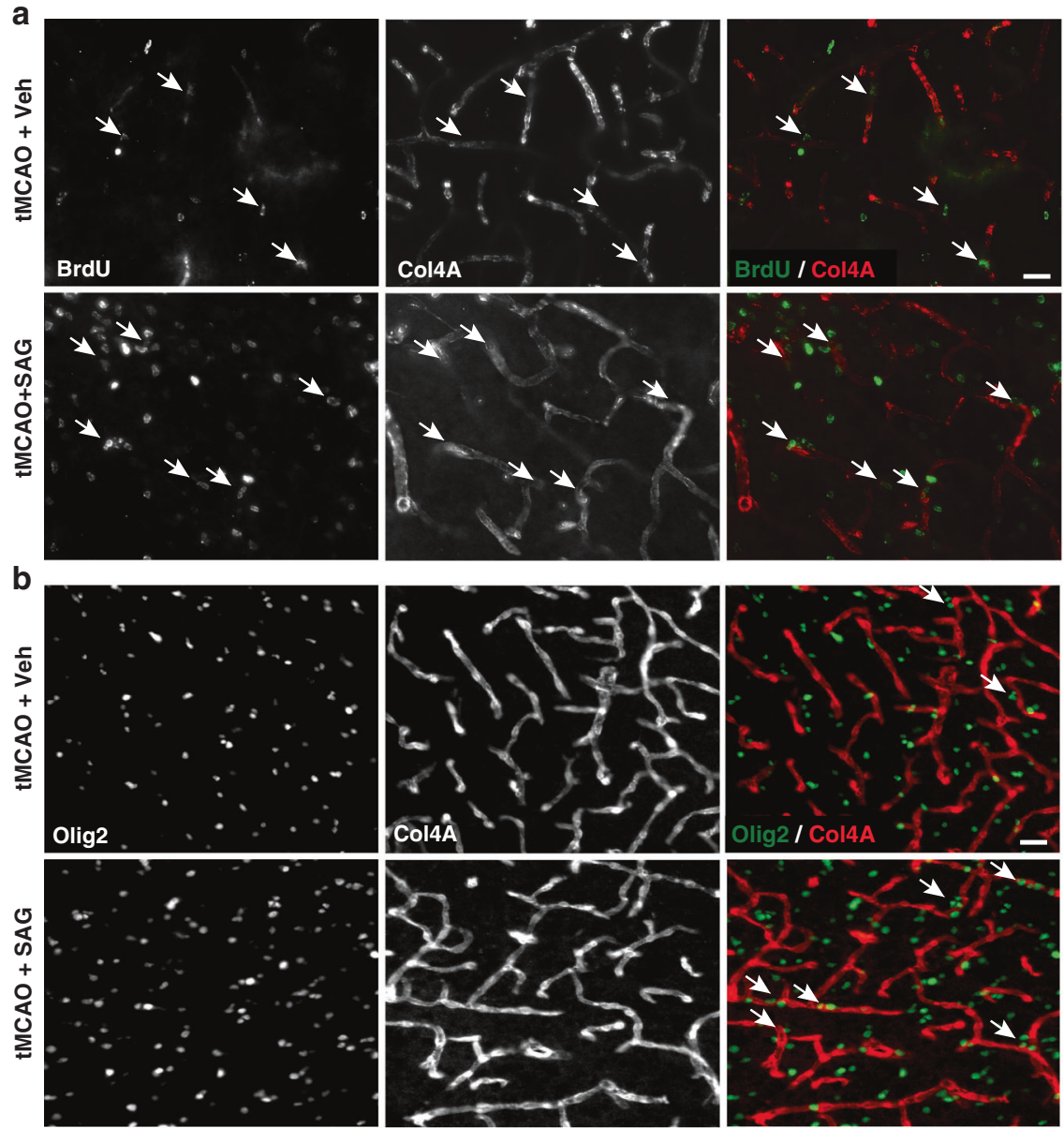

C
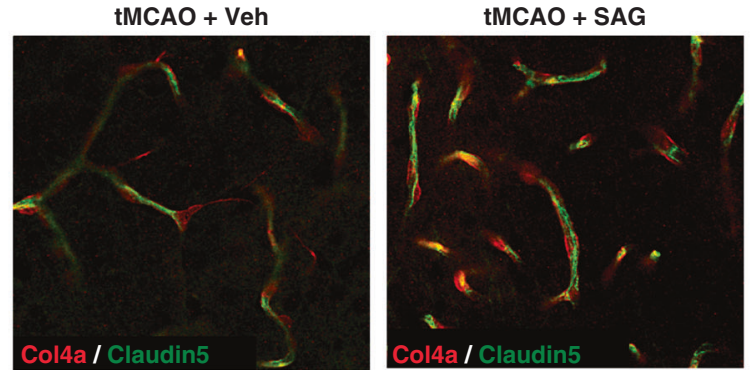

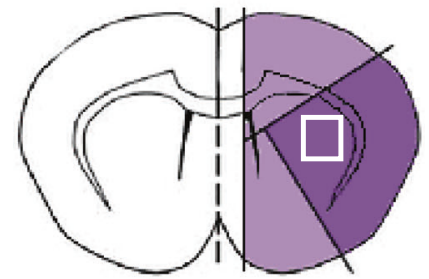

d

Co-labeled BrdU+/Col4A+

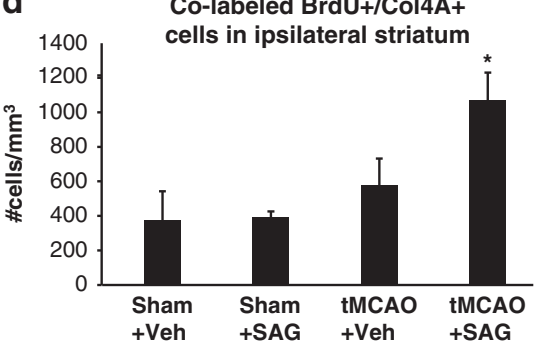

e
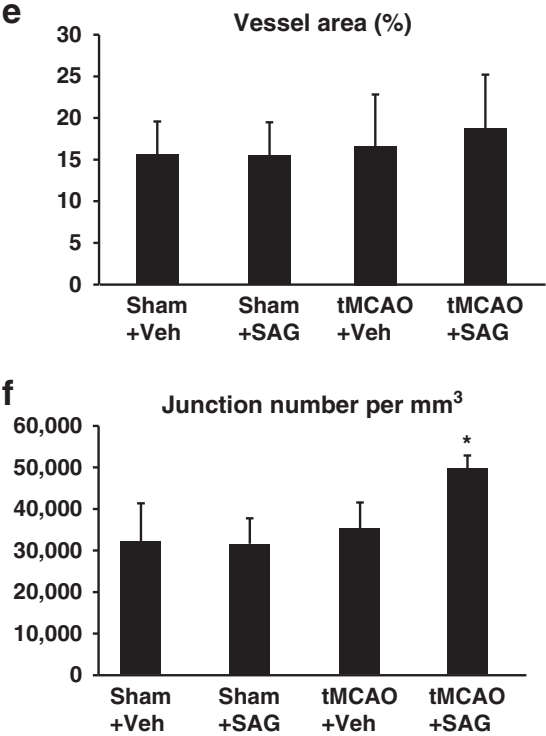

Fig. 5 SAG enhances angiogenesis following tMCAO. a Cells in the injured core (striatum) stained with BrdU and Collagen 4A (Col4A), an EC marker, to visualize proliferation of cells associated with the vasculature. Arrows denote BrdU+ cells in direct contact with Col4A+ vessels. Scale bar, $100 \mu \mathrm{m}$. Right, diagram depicting area of analysis. b Cells co-stained with Olig2 and Col4A in the striatum. Arrows denote Olig2+ cells in contact with Col4A+ vessels. c Cells co-stained with Col4A and Claudin5, a marker of blood-brain barrier (BBB) structure. d Quantification of BrdU+/Col4A + cells in the ipsilateral striatum per $\mathrm{mm}^{3}$. e Quantification of vessel area from Col4A-labeled vasculature in the motor and somatosensory cortical regions bordering the striatal core. $\mathbf{f}$ Quantification of vessel junction number per mm ${ }^{3}$ in the same region as e. ${ }^{*} p<0.05$, one-way ANOVA with Tukey's multiple comparisons test.

rodent models of neonatal cerebellar injury, Down syndrome, ${ }^{8}$ and adult stroke. ${ }^{13}$ Here we extended these findings to a rat model of NS and report the novel finding that a single dose of SAG has beneficial effects to preserve brain volume, decrease gliosis, increase endothelial and oligodendroglial lineage proliferation, and enhance long-term behavioral outcomes.

SAG promotes oligodendroglial and endothelial proliferation after brain injury

We examined the effect of post-injury SAG administration on oligodendroglial lineage in two well-established preclinical brain injury rodent models, tMCAO NS to examine the effects in developing brain injury and EB-induced focal demyelination in adult rats to focus on the oligodendroglial response. ${ }^{12}$ There was a proliferative oligodendroglial response with SAG treatment in both models. Recent work has shown that increased proliferation is a prerequisite for the oligodendroglial differentiation ${ }^{31}$ and that newly generated oligodendrocytes exhibit enhanced and accurate axonal myelination. ${ }^{32}$ Although we did not see a significant increase in mature $\mathrm{CC} 1+$ cells at these early time points, further studies are needed to determine whether SAG-induced oligodendroglial proliferation might benefit myelin generation in the long term.

Angiogenesis is critical for long-term repair following early focal ischemia-reperfusion injury, but remarkably little is known regarding the local vascular response to early focal ischemia-reperfusion injury 
a

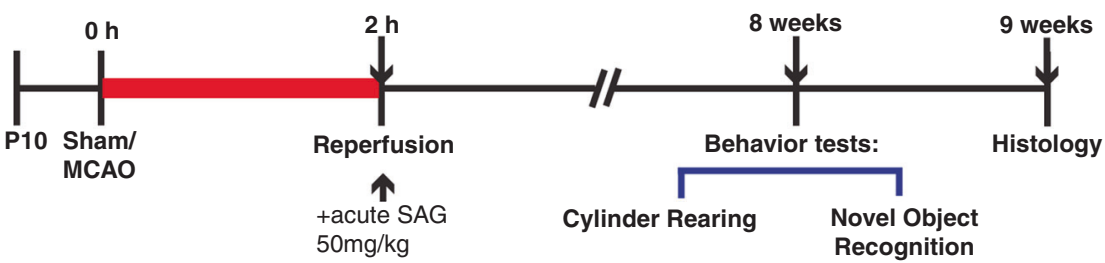

b

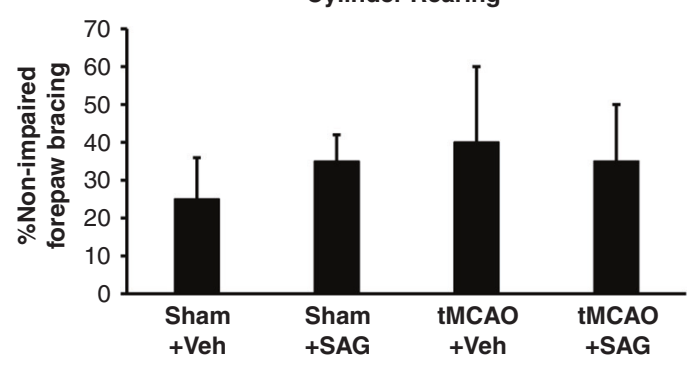

d

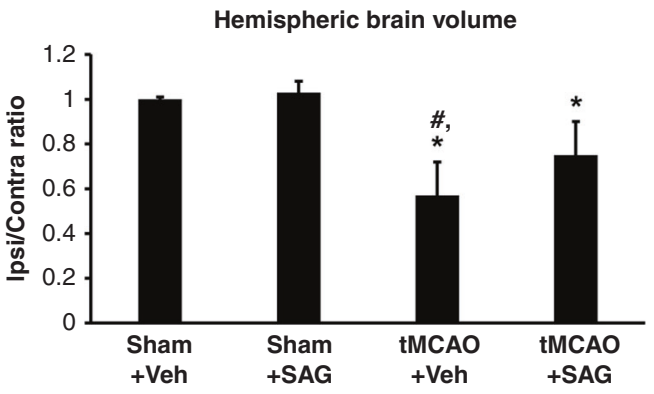

f

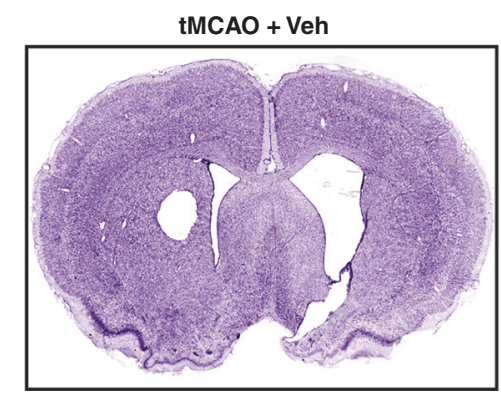

C

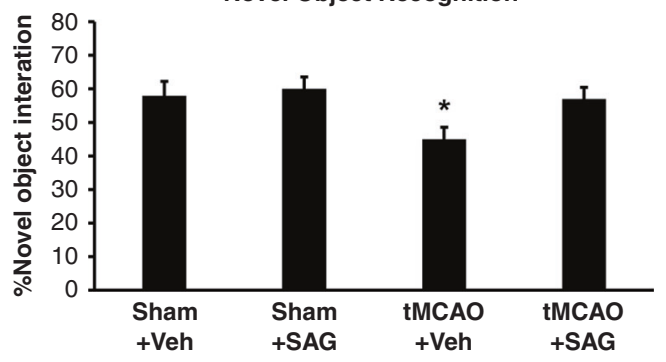

e $1.21 \quad$ Corpus callosum volume

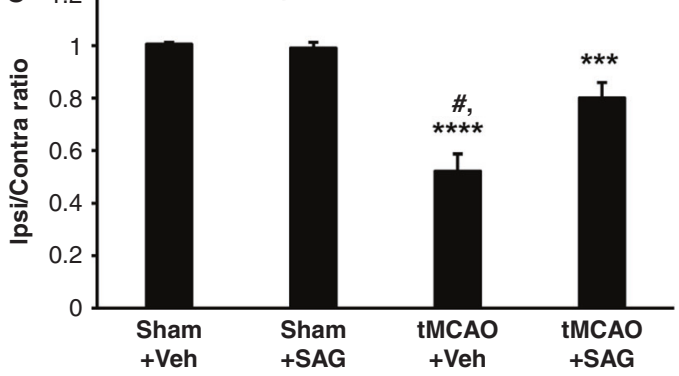

tMCAO + SAG

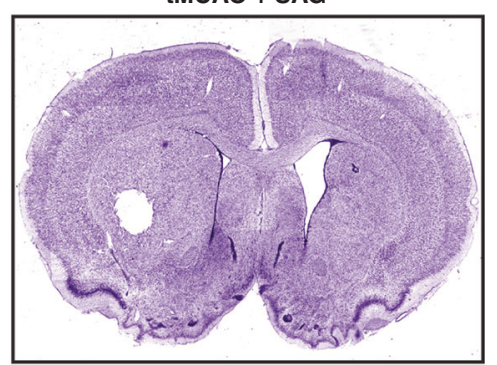

Fig. 6 SAG shows improvements in behavioral tests. a Experimental timeline for surgery, treatment, and behavioral tests for long-term neurobehavioral outcome. $\mathbf{b}$ Quantification of gross sensorimotor function between groups as assessed by forelimb preference in the Cylinder Rearing test. c Quantification of Novel Object Recognition test. d Quantification of ipsilateral-to-contralateral ratio of hemispheric brain volume. e Quantification of ipsilateral-to-contralateral ratio of corpus callosum volume. $\mathbf{f}$ Representative images of coronal sections from rat brains 2 months post-tMCAO or sham surgery. ${ }^{*} p<0.05,{ }^{* * *} p<0.001,{ }^{* * *} p<0.0001$ vs Sham +Veh; $p<0.05$ vs tMCAO+SAG, one-way ANOVA with Tukey's multiple comparisons test.

in the brain..$^{33}$ In an adult stroke model, Shh administration increased microvessel density and EC number 7 days after $\mathrm{MCAO}^{13}$ while Smo agonist cyclopamine blocked neuroprotective effects in early subarachnoid hemorrhage. ${ }^{34}$ In our model of neonatal tMCAO, SAG administration promoted OPC numbers and angiogenesis. We found newly generated ECs in the injured core coupled with increased vascular branching after SAG treatment, suggesting enhanced vascular remodeling. Although we note that SAG did not regulate endothelial tight junction based on Claudin 5 expression, we do not rule out SAG affecting BBB permeability. Future extravasation studies at different time points will provide insight into this.

In the developing embryonic and postnatal brain, the vasculature engages in close interactions with migratory OPCs in both mice and humans ${ }^{24}$ likely involving Wnt signaling during development and the response to injury. ${ }^{4,24}$ In NS, we observed increased numbers of oligodendroglial cells in close apposition to blood vessels near the injured core in SAG-treated animals. Whether SAG directly promotes vascular remodeling or is a secondary consequence of oligodendrogenesis remains to be elucidated.

One-time SAG treatment preserved brain volume and improved behavioral outcomes long term after neonatal brain injury We found that a single dose of SAG improved long-term behavioral outcomes and brain volume in a model of NS. NOR testing showed that treated animals had improved recognition memory of objects over a length of time. In contrast, we did not see long-term changes in sensorimotor function at young adulthood by $C R$; however, there was significant variability within groups, and this test may not adequately analyze fine motor 
deficits at this time point following early injury. In addition, SAG treatment significantly preserved brain volume indicating that it has both short- and long-term benefit in a model of NS.

Potential therapeutic benefits of SAG in NS

NS constitutes a major health problem in the United States and worldwide, with the majority of survivors exhibiting long-term neurodevelopmental deficits. ${ }^{35,36}$ There are currently no accepted small molecule neuroprotective interventions for NS, and such therapies could be useful in acute settings outside major centers that provide hypothermia, including low- and middle-income countries.

Investigation of new treatment strategies for NS should take into account both etiology and timing of injury to be as relevant as possible to human newborn injury and thus translation to the clinic. ${ }^{37}$ While erythropoietin has demonstrated consistent benefit with post-injury treatment in different models of early brain injury, including stroke, $^{38}$ we note that multiple doses must be administered and that single-dose therapy does not confer longterm benefits. $^{39}$ In contrast, our findings demonstrate clear benefits of one-time treatment with SAG following tMCAO. However, as we have measured SAG bioactivity duration in a transgenic mouse model and not directly in our rat model, we acknowledge that species differences may result in differential responses. In addition, we note that prolonged bioactivity does not imply that a single dose is either sufficient or most beneficial. Future work will examine alternative treatment plan, such as multiple doses or delayed therapy.

Mounting evidence suggests that SAG is neuroprotective against injuries affecting preterm and term human neonates. We found that SAG preserves brain volume and increases OPC number, vascular proliferation, and long-term histological and cognitive benefit with a single dose given immediately post-NS. Therefore, this study supports investigation of SAG efficacy in larger animal models and further translational development with a view to ultimate testing in human clinical trials.

\section{ACKNOWLEDGEMENTS}

We would like to thank members of the Gonzalez, Franklin, and Rowitch laboratories for suggestions and technical assistance with experimental set-up. M.C. acknowledges fellowship awards from the American Heart Association and The Children's Heart Foundation and funding support from a Career Development Grant awarded by Cerebral Palsy Alliance Research Foundation. This work was supported by funding from the Harrington Discovery Institute (to D.H.R.), UK MS Society (to R.J.M.F.), the Adelson Medical Research Foundation (to D.H.R. and R.J.M.F.), and the National Institutes of Health, NINDS (P01-NS083513 to D.H.R., K99-NS117804 to M.C., R01NS107039 and U01-NS092764 to F.G.). This research was also funded by the Wellcome Trust (to D.H.R.) and supported by the NIHR Cambridge Biomedical Research Centre.

\section{AUTHOR CONTRIBUTIONS}

V.N., M.C., A.L., S.K. and G.G. performed experimental procedures. V.N., D.H.R. and F.G. designed research and planned all the experiments. V.N., M.C., S.K., G.G. and F.G. analyzed the data and prepared the figures. V.N., R.J.M.F., D.H.R. and F.G. wrote the article. D.H.R. and F.G. conceived and led the project. All the authors read and approved the final version of the paper.

\section{ADDITIONAL INFORMATION}

Supplementary information The online version contains supplementary material available at https://doi.org/10.1038/s41390-021-01408-7.

Competing interests: The authors declare no competing interests.

Patient consent: Patient consent was not required for this study.

Publisher's note Springer Nature remains neutral with regard to jurisdictional claims in published maps and institutional affiliations.

\section{REFERENCES}

1. Grunt, S. et al. Incidence and outcomes of symptomatic neonatal arterial ischemic stroke. Pediatrics 135, e1220-e1228 (2015).

2. Dunbar, M. \& Kirton, A. Perinatal stroke. Semin Pediatr. Neurol. 32, 100767 (2019).

3. van Tilborg, E. et al. Origin and dynamics of oligodendrocytes in the developing brain: Implications for perinatal white matter injury. Glia 66, 221-238 (2018).

4. Yuen, T. J. et al. Oligodendrocyte-encoded HIF function couples postnatal myelination and white matter angiogenesis. Cell 158, 383-396 (2014).

5. Falcon-Urrutia, P., Carrasco, C. M., Lois, P., Palma, V. \& Roth, A. D. Shh signaling through the primary cilium modulates rat oligodendrocyte differentiation. PLOS ONE 10, e0133567 (2015).

6. Alvarez, J. I. et al. The Hedgehog pathway promotes blood-brain barrier integrity and CNS immune quiescence. Science 334, 1727-1731 (2011).

7. Heine, V. M. et al. A small-molecule smoothened agonist prevents glucocorticoidinduced neonatal cerebellar injury. Sci. Transl. Med. 3, 105ra104 (2011).

8. Das, I. et al. Hedgehog agonist therapy corrects structural and cognitive deficits in a Down syndrome mouse model. Sci. Transl. Med. 5, 201 ra120 (2013).

9. Gonzalez, F. F. et al. Erythropoietin increases neurogenesis and oligodendrogliosis of subventricular zone precursor cells after neonatal stroke. Stroke 44, 753-758 (2013).

10. Smith, K. J., Blakemore, W. F. \& McDonald, W. I. Central remyelination restores secure conduction. Nature 280, 395-396 (1979).

11. Fancy, S. P. et al. Axin2 as regulatory and therapeutic target in newborn brain injury and remyelination. Nat. Neurosci. 14, 1009-1016 (2011).

12. Woodruff, R. H. \& Franklin, R. J. Demyelination and remyelination of the caudal cerebellar peduncle of adult rats following stereotaxic injections of lysolecithin, ethidium bromide, and complement/anti-galactocerebroside: a comparative study. Glia 25, 216-228 (1999).

13. Chen, S. C. et al. Administration of Sonic hedgehog protein induces angiogenesis and has therapeutic effects after stroke in rats. Neuroscience 352, 285-295 (2017).

14. Chen, J. K., Taipale, J., Young, K. E., Maiti, T. \& Beachy, P. A. Small molecule modulation of Smoothened activity. Proc. Natl Acad. Sci. USA 99, 14071-14076 (2002).

15. Becher, O. J. et al. Gli activity correlates with tumor grade in platelet-derived growth factor-induced gliomas. Cancer Res. 68, 2241-2249 (2008).

16. Larpthaveesarp, A., Georgevits, M., Ferriero, D. M. \& Gonzalez, F. F. Delayed erythropoietin therapy improves histological and behavioral outcomes after transient neonatal stroke. Neurobiol. Dis. 93, 57-63 (2016).

17. Franklin, R. J. \& Ffrench-Constant, C. Remyelination in the CNS: from biology to therapy. Nat. Rev. Neurosci. 9, 839-855 (2008).

18. Sim, F. J., Zhao, C., Penderis, J. \& Franklin, R. J. The age-related decrease in CNS remyelination efficiency is attributable to an impairment of both oligodendrocyte progenitor recruitment and differentiation. J. Neurosci. 22, 2451-2459 (2002).

19. Griffiths, I. R., Schneider, A., Anderson, J. \& Nave, K. A. Transgenic and natural mouse models of proteolipid protein (PLP)-related dysmyelination and demyelination. Brain Pathol. 5, 275-281 (1995).

20. Grow, J. L., Liu, Y. Q. \& Barks, J. D. Can lateralizing sensorimotor deficits be identified after neonatal cerebral hypoxia-ischemia in rats? Dev. Neurosci. 25 394-402 (2003).

21. Huang, T.-N. \& Hsueh, Y.-P. Novel object recognition for studying memory in mice. Bio-protocol 4, e1249 (2014).

22. Regeur, L. \& Pakkenberg, B. Optimizing sampling designs for volume measurements of components of human brain using a stereological method. J. Microsc. 155, 113-121 (1989)

23. Galinsky, R. et al. Complex interactions between hypoxia-ischemia and inflammation in preterm brain injury. Dev. Med. Child Neurol. 60, 126-133 (2018).

24. Tsai, H. H. et al. Oligodendrocyte precursors migrate along vasculature in the developing nervous system. Science 351, 379-384 (2016).

25. Jin, Y., Barnett, A., Zhang, Y., Yu, X. \& Luo, Y. Poststroke Sonic hedgehog agonist treatment improves functional recovery by enhancing neurogenesis and angiogenesis. Stroke 48, 1636-1645 (2017).

26. Alvarez-Buylla, A. \& Ihrie, R. A. Sonic hedgehog signaling in the postnatal brain Semin. Cell Dev. Biol. 33, 105-111 (2014).

27. Wechsler-Reya, R. J. \& Scott, M. P. Control of neuronal precursor proliferation in the cerebellum by Sonic hedgehog. Neuron 22, 103-114 (1999).

28. Garcia, A. D. R. et al. The elegance of Sonic hedgehog: emerging novel functions for a classic morphogen. J. Neurosci. 38, 9338-9345 (2018).

29. Chapouly, C., Guimbal, S. Hollier, P. L. \& Renault, M.-A. Role of hedgehog signaling in vasculature development, differentiation, and maintenance. Int. J. Mol. Sci. 20, 3076 (2019).

30. Winkler, C. C. et al. The dorsal wave of neocortical oligodendrogenesis begins embryonically and requires multiple sources of Sonic hedgehog. J. Neurosci. $\mathbf{3 8}$ 5237-5250 (2018).

31. Foerster, S. et al. Proliferation is a requirement for differentiation of oligodendrocyte progenitor cells during CNS remyelination. Preprint at bioRxiv https://doi. org/10.1101/2020.05.21.108373 (2020). 
32. Neely, S. A. et al. New oligodendrocytes exhibit more abundant and accurate myelin regeneration than those that survive demyelination. Preprint at bioRxiv https://doi.org/10.1101/2020.05.22.110551 (2020).

33. Baburamani, A. A., Ek, C. J., Walker, D. W. \& Castillo-Melendez, M. Vulnerability of the developing brain to hypoxic-ischemic damage: contribution of the cerebral vasculature to injury and repair? Front. Physiol. 3, 424 (2012).

34. $\mathrm{Hu}, \mathrm{Q}$. et al. Neuroprotective effects of a smoothened receptor agonist against early brain injury after experimental subarachnoid hemorrhage in rats. Front. Cell. Neurosci. 10, 306 (2016).

35. Kirton, A. \& deVeber, G. Paediatric stroke: pressing issues and promising directions. Lancet Neurol. 14, 92-102 (2015).

36. Dunbar, M. et al. Population based birth prevalence of disease-specific perinatal stroke. Pediatrics 146, e2020013201 (2020).

37. Wernig, M. et al. Neurons derived from reprogrammed fibroblasts functionally integrate into the fetal brain and improve symptoms of rats with Parkinson's disease. Proc. Natl Acad. Sci. USA 105, 5856-5861 (2008).

38. Traudt, C. M. \& Juul, S. E. Erythropoietin as a neuroprotectant for neonatal brain injury: animal models. Methods Mol. Biol. 982, 113-126 (2013).
39. Gonzalez, F. F. et al. Erythropoietin sustains cognitive function and brain volume after neonatal stroke. Dev. Neurosci. 31, 403-411 (2009).

(i) Open Access This article is licensed under a Creative Commons Attribution 4.0 International License, which permits use, sharing, adaptation, distribution and reproduction in any medium or format, as long as you give appropriate credit to the original author(s) and the source, provide a link to the Creative Commons license, and indicate if changes were made. The images or other third party material in this article are included in the article's Creative Commons license, unless indicated otherwise in a credit line to the material. If material is not included in the article's Creative Commons license and your intended use is not permitted by statutory regulation or exceeds the permitted use, you will need to obtain permission directly from the copyright holder. To view a copy of this license, visit http://creativecommons. org/licenses/by/4.0/.

(c) The Author(s) 2021 